\title{
High-Frequency Oscillatory Ventilation (HFOV) and Airway Pressure Release Ventilation (APRV): A Practical Guide
}

Journal of Intensive Care Medicine Volume 24 Number 4 July/August 2009 215-229 (C) 2009 SAGE Publications $10.1177 / 0885066609335728$ http://jicm.sagepub.com hosted at http://online.sagepub.com

\section{S. P. Stawicki, MD, Munish Goyal, MD, and Babak Sarani, MD, FACS}

Despite advances in ventilator management, $31 \%$ to $38 \%$ of patients with adult respiratory distress syndrome (ARDS) will die, some from progressive respiratory failure. Inability to adequately oxygenate patients with severe ARDS has prompted extensive efforts to identify what are now known as alternative modes of ventilation including high-frequency oscillatory ventilation and airway pressure release ventilation. Both modalities are based on the principles of the open-lung concept and aim to improve oxygenation by keeping the lung uniformly inflated for an extended period of time.
Although a mortality benefit has not been proven, some patients may benefit from these alternative modes of ventilation as rescue measures while the underlying process resolves. The purpose of this article is to review the evidence and mechanisms underlying each modality and to describe the fundamental steps in initiating, adjusting, and terminating these modes of ventilation.

Keywords: alternative mechanical ventilation; open lung; APRV; HFOV

\section{Introduction}

Inability to adequately oxygenate patients with acute lung injury (ALI) or adult respiratory distress syndrome (ARDS) has prompted extensive efforts to identify what are now known as alternative modes of ventilation. Animal studies demonstrate that mechanical ventilation with large tidal volumes and high airway pressures leads to severe alterations in permeability, pulmonary edema, and diffuse alveolar damage similar to the pathologic findings characteristic of ARDS. ${ }^{1,2}$ Mechanisms thought to be involved in ventilator-induced lung injury (VILI) include

From the Division of Traumatology and Surgical Critical Care, Departments of Surgery (SPS, MG, BS) and Emergency Medicine (MG), University of Pennsylvania School of Medicine, Philadelphia, Pennsylvania.

Received October 29, 2007, and in revised form April 28, 2008. Accepted for publication May 05, 2008.

Address correspondence to: Babak Sarani, 3440 Market Street, First Floor, Philadelphia, PA 19104; e-mail: saranib@uphs. upenn.edu. excessive stretch of the alveoli (volutrauma), shear injury due to repetitive alveolar collapse and reopening (atelectrauma), and excessive pressure within the alveoli (barotrauma).

Application of low tidal volume/low plateau pressure strategies during conventional ventilation in adults with ALI or ARDS results in decreased duration of mechanical ventilation and mortality relative to high tidal volume/high plateau pressure ventilation. ${ }^{3,4}$ However, $31 \%$ to $38 \%$ of patients proceed to die, some from progressive respiratory failure, indicating that conventional ventilation with lung protective strategies may not be adequate. ${ }^{3,4}$ Although a mortality benefit has not been proven, such patients may benefit from alternative modes of ventilation as rescue measures to maintain oxygenation while the underlying process resolves.

High-frequency oscillatory ventilation (HFOV) and airway pressure release ventilation (APRV) are 2 commonly used alternative modes of mechanical ventilation in this patient population. Both modalities are based on the principles of the open-lung concept (OLC) and aim to improve oxygenation by 

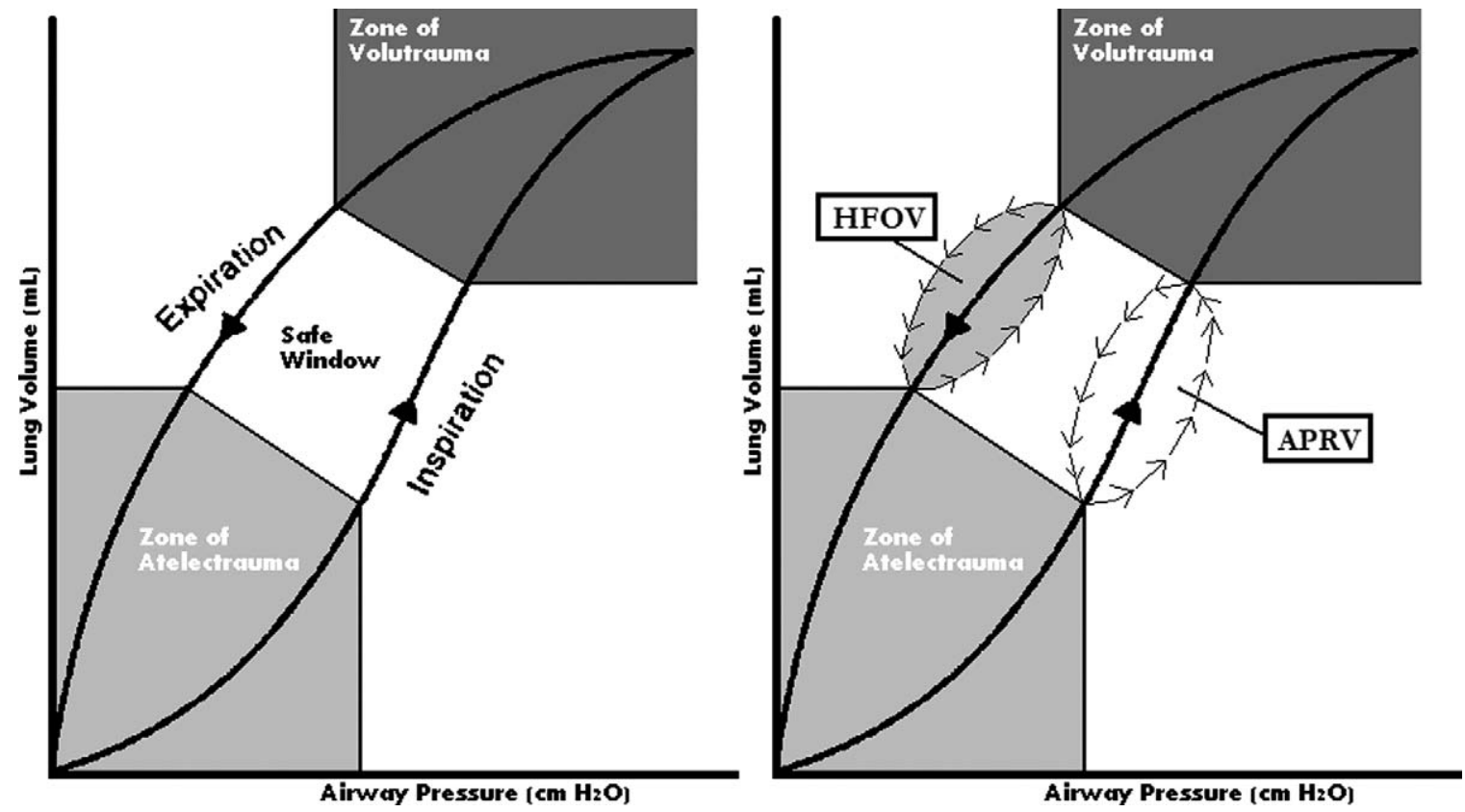

Figure 1. Pressure-volume curve comparing regions of ventilation using high-frequency oscillatory ventilation (HFOV) and airway pressure release ventilation (APRV). Note that ventilation with HFOV occurs on the expiratory limb, whereas ventilation with APRV occurs on the inspiratory limb of the curves.

keeping the lung uniformly inflated for an extended period of time. The OLC was originally coined by Lachmann in 1992 and highlights the importance of maintaining open alveoli while minimizing overdistension and atelectrauma. ${ }^{5}$ In general, either mode could be considered when the fraction of inspired oxygen $\left(\mathrm{FiO}_{2}\right)$ is greater than $60 \%$, positive end-expiratory pressure (PEEP) is greater than $15 \mathrm{~cm} \mathrm{H}_{2} \mathrm{O}$, plateau pressure is greater than $30 \mathrm{~cm}$ $\mathrm{H}_{2} \mathrm{O}$, and the arterial oxygen saturation is less than $90 \%{ }^{6}$

The purpose of this article is to review the evidence and mechanisms underlying each modality and to describe the fundamental steps in initiating, adjusting, and terminating these modes of ventilation. Discussion is limited to adult patients. It is assumed that the reader has a sound understanding of the pathophysiology of ARDS and VILI.

\section{High-Frequency Oscillatory Ventilation}

High-frequency oscillatory ventilation has been identified as an alternative method of applying low tidal volume, controlled pressure ventilation in the setting of ARDS. ${ }^{7}$ Traditionally used with great success in neonatology, HFOV has recently been recognized as potentially useful in adult patients with ARDS. ${ }^{7}$ Its use in adults is based on the hope that it will improve oxygenation without further injuring the lung. The only HFOV approved for use in adults is the Sensormedics 3100B (Viasys Healthcare, Yorba Linda, CA).

\section{Rationale Behind HFOV}

Patients who develop ARDS or ALI have reduced lung compliance and impaired oxygenation. ${ }^{8}$ Highfrequency oscillatory ventilation attempts to deal with potential risks of mechanical ventilation, barotrauma, volutrauma, atelectrauma, and oxygen toxicity and can be considered when conventional ventilation fails to safely and adequately provide respiratory support. High frequency ventilation is generally considered beneficial for patients with severe pulmonary failure because $(a)$ it uses much smaller tidal volumes than conventional ventilation, (b) it maintains the lungs/alveoli open on the deflation limb of the pressure-volume curve (Figure 1) at a relatively constant airway pressure and thus may prevent atelectrauma and barotrauma, ${ }^{9}$ and $(c)$ it improves ventilation/perfusion (V/Q) matching by ensuring uniform aeration of the lung. ${ }^{9}$ It is possible 
Table 1. Suggested Initial Settings for APRV and HFOV

\begin{tabular}{|c|c|c|c|}
\hline \multicolumn{2}{|l|}{$\mathrm{HFOV}^{10}$} & \multicolumn{2}{|r|}{ APRV } \\
\hline Frequency & & $T_{\text {high }}$ & $4-6$ seconds \\
\hline $\mathrm{pH}<7.1$ & $4 \mathrm{~Hz}$ & $\mathrm{~T}_{\text {low }}$ & $0.6-0.8$ seconds based on T-PEFR ${ }^{a}$ \\
\hline $\mathrm{pH} 7.1-7.19$ & $5 \mathrm{~Hz}$ & $P_{\text {high }}$ & $\begin{array}{l}\text { Same as plateau pressure on } \mathrm{CV} \text { or } \\
\mathrm{P}_{\mathrm{aw}}+2-4 \mathrm{~cm} \mathrm{H}_{2} \mathrm{O} \text { if transition from HFOV }\end{array}$ \\
\hline $\mathrm{pH} 7.2-7.35$ & $6 \mathrm{~Hz}$ & $P_{\text {low }}$ & 0 \\
\hline $\mathrm{pH}>7.35$ & $7 \mathrm{~Hz}$ & $\mathrm{FiO}_{2}$ & $100 \%$ \\
\hline Amplitude (power) & $70-90 \mathrm{~cm} \mathrm{H}_{2} \mathrm{O}$ & & \\
\hline $\mathrm{P}_{\mathrm{aw}}$ & $\begin{array}{l}5 \mathrm{~cm} \mathrm{H}_{2} \mathrm{O}>\text { plateau pressure on } \\
\mathrm{CV} \text { to max of } 35 \mathrm{~cm} \mathrm{H}_{2} \mathrm{O}\end{array}$ & & \\
\hline Bias flow & $40 \mathrm{~L} / \mathrm{min}$ & & \\
\hline Inspiratory time & $33 \%$ & & \\
\hline $\mathrm{FiO}_{2}$ & $100 \%$ & & \\
\hline
\end{tabular}

NOTES: APRV = airway pressure release ventilation; $\mathrm{CV}=$ conventional ventilation; $\mathrm{HFOV}=$ high-frequency oscillatory ventilation; $\mathrm{P}_{\mathrm{aw}}$, mean airway pressure.

${ }^{\mathrm{a}} \mathrm{T}_{\text {low }}$ may have to be greater than 1 second in patients with severe obstructive lung disease.

that adults with severe ARDS may benefit from HFOV due to the long and variable time constant required for filling of noncompliant alveoli. Such alveoli may experience atelectrauma even with low tidal volume, moderate PEEP conventional ventilation. Similarly, less fibrotic segments of the lung may experience cyclic volutrauma due to preferential air flow into these segments with conventional ventilation. Various studies have used different criteria for determining when HFOV should be instituted in adults, but a consensus article that sought to adhere to lung protective strategies of mechanical ventilation while maintaining adequate gas exchange, suggested its use when conventional ventilator settings require an $\mathrm{FiO}_{2}$ greater than $70 \%$ and PEEP greater than $14 \mathrm{~cm} \mathrm{H}_{2} \mathrm{O}$ or when the arterial $\mathrm{pH}$ is less than 7.25 with a tidal volume that is greater than $6 \mathrm{~cm}^{3} / \mathrm{kg}$ and a plateau pressure that is greater than $30 \mathrm{~cm} \mathrm{H}_{2} \mathrm{O}{ }^{10}$

Several studies in adults have shown that oxygenation improves after implementation of HFOV, but mortality benefit has not been demonstrated in randomized trials. ${ }^{11,9,12-14}$ However, these studies were not powered sufficiently to detect small changes in mortality. In a study by Fort et al, ${ }^{9} \mathrm{HFOV}$ was evaluated in terms of safety and effectiveness in patients with ARDS. ${ }^{9}$ This prospective study included patients with mean peak inspiratory pressure of $54.3 \pm 12.7 \mathrm{~cm} \mathrm{H} \mathrm{H}_{2} \mathrm{O}, \mathrm{PaO}_{2} / \mathrm{FiO}_{2}$ ratio of $68.6 \pm 21.6$, and PEEP of $18.2 \pm 6.9 \mathrm{~cm} \mathrm{H}_{2} \mathrm{O}$. High-frequency oscillatory ventilation was instituted after varying periods of conventional ventilation $(5.12 \pm 4.3$ days). A lung volume recruitment strategy was used concurrently. During the study, $76 \%$ of patients demonstrated improved gas exchange and an overall improvement in $\mathrm{PaO}_{2} / \mathrm{FiO}_{2}$ ratio. Cardiac output was not compromised in any of the patients despite increases in mean airway pressure, lending credence to the fact that HFOV is both a safe and an effective means to augment oxygenation in adult patients with severe ARDS failing conventional ventilation. Thus, patients who need maximal alveolar recruitment to keep the $\mathrm{FiO}_{2}$ below toxic levels may benefit from HFOV.

\section{Mechanics of High-Frequency Oscillatory Ventilation}

The variables that are controlled directly on the $3100 \mathrm{~B}$ ventilator are respiratory frequency, amplitude of ventilation (also called the power or $\Delta \mathrm{P}$ ), mean airway pressure $\left(\mathrm{P}_{\mathrm{aw}}\right)$, bias gas flow rate, percentage of inspiratory time, and $\mathrm{FiO}_{2}$. Suggested initial ventilator settings are noted in Table 1 . The core of the HFOV system consists of a piston assembly that incorporates an electronic control circuit, or square-wave driver, which powers a linear drive motor (Figure 2). This motor consists of an electrical coil within a magnet, similar to a permanent magnet speaker. When a positive polarity is applied to the square-wave driver, the coil is driven forward. The coil is attached to a rubber bellows, or diaphragm, to create a piston. When the coil moves forward, the piston moves forward, resulting in the creation of the inspiratory phase. When the polarity becomes negative, the electrical coil and the attached piston are 


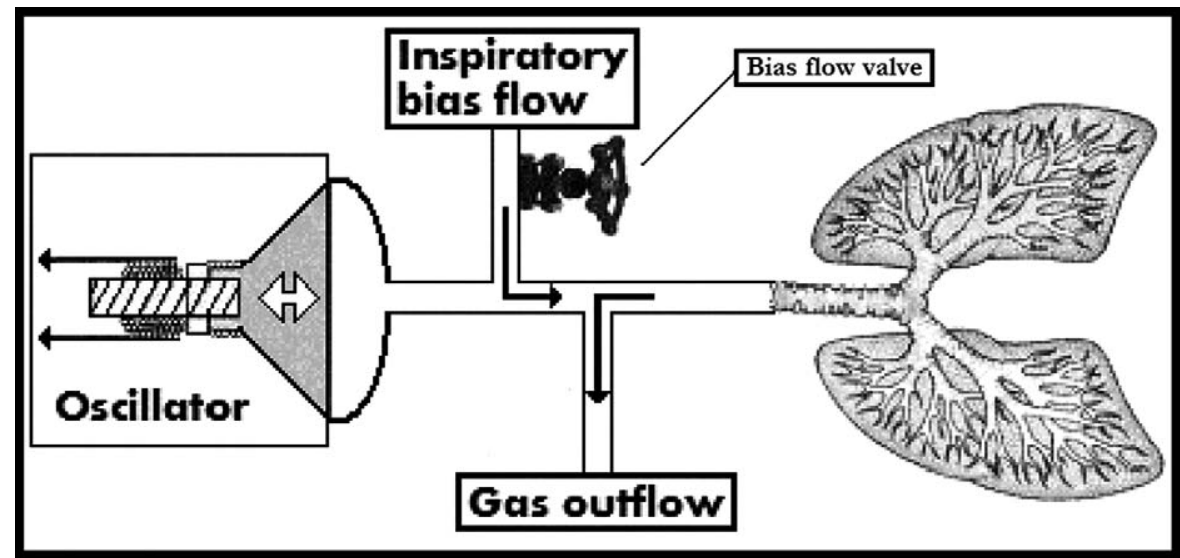

Figure 2. Basic design of the high frequency oscillating ventilator. A bias gas flow is moved rapidly by a piston-driven assembly.

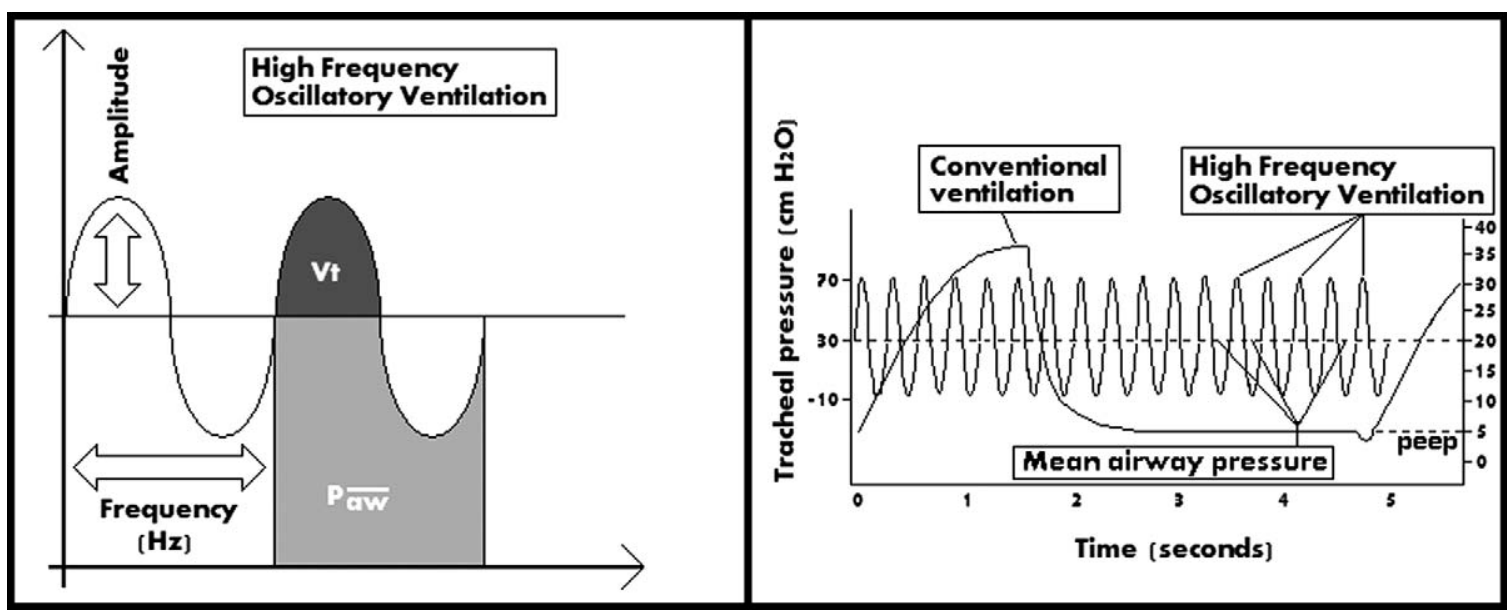

Figure 3. Waveforms depicting the key variables that are controlled during high frequency oscillation as compared to conventional ventilation. The y-axis on the left depicts changes in airway pressure seen with high frequency oscillatory ventilation and the y-axis on the right depicts changes in peak airway pressure with conventional ventilation. Note that tracheal pressure becomes negative at peak expiration, thereby making expiration an active process. Also note that as amplitude increases, delivered minute ventilation increases. A background tracing of pressure versus time using a respiratory rate of 12 and inspiratory to expiratory ratio of 1:3 with conventional ventilation is presented for comparison.

driven away from the patient, creating the expiratory phase. By moving rapidly, the diaphragm oscillates a constant stream of gas, called the bias gas flow, through the airways. It is recommended that the lung be aggressively recruited prior to the start of HFOV to ensure that all the potentially recruitable alveoli are open.

The speed of oscillation is set by manipulating the frequency (Figure 3). One Hertz is equal to 1 breath per second, that is, 60 breaths per minute. A frequency of $5 \mathrm{~Hz}$ gives a frequency of 5 breaths per second or 300 breaths per minute. An important point to remember is that given a fixed inspiratory to expiratory time ratio, as frequency is increased, the excursion of the piston is limited by the time allocated for each breath cycle. Therefore, changes in frequency are inversely proportional to the amplitude and thus delivered tidal volume (see below). Alveoli with short-time constants for air flow (high lung compliance and low airway resistance) can be ventilated more effectively at higher frequencies than those which have longer time constants (low lung compliance or high airway resistance). Frequency selection also directly affects the pressure cycles 
Table 2. Ventilator Adjustments Based on Blood Gas Results

\section{HFOV}

APRV

Hypoxemia

- Increase $\mathrm{FiO}_{2}$

- Increase $\mathrm{P}_{\mathrm{aw}}$ by $2 \mathrm{~cm} \mathrm{H}_{2} \mathrm{O}$ to $\max 40 \mathrm{~cm} \mathrm{H} \mathrm{H}_{2} \mathrm{O}$

- Recruitment maneuver in cases of recurrent hypoxemia

- Increase bias flow

Hypercapnea/acidemia

- Decrease frequency to nadir of $3 \mathrm{~Hz}$

- Increase power to max $90 \mathrm{~cm} \mathrm{H}_{2} \mathrm{O}$

- Introduce endotracheal cuff leak

- Increase $\mathrm{FiO}_{2}$

- Recruitment maneuver

- Increase $\mathrm{T}_{\text {high }}$

- Increase $\mathrm{P}_{\text {high }}$ to $\max 40 \mathrm{~cm} \mathrm{H}_{2} \mathrm{O}$

- Adjust $\mathrm{T}_{\text {low }}$ to keep T-PEFR $>50 \%$

- Ensure patient is spontaneously breathing

- Increase $\mathrm{T}_{\text {low }}$

- Increase $\mathrm{P}_{\text {high }}$ and $\mathrm{T}_{\text {high }}$ to increase minute ventilation

NOTES: APRV = airway pressure release ventilation; HFOV $=$ high-frequency oscillatory ventilation; $\mathrm{FiO}_{2}=\mathrm{Fraction}_{\mathrm{of}}$ inspired oxygen; $\mathrm{P}_{\mathrm{aw}}=$ Mean Airway Pressure; T-PEFR = Terminal peak expiratory flow rate.

applied to the lung. A smaller percentage of the circuit change in pressure is transmitted at higher frequencies. Whereas the normal lung can be ventilated over a wide pressure range without inducing injury, the lung with poor compliance has a very limited zone of safety. ${ }^{15}$ As frequency increases, the zone of safe pressure widens, making it easier to maintain more of the lung homogenously aerated. ${ }^{15}$ Unfortunately, there is no simple formula for estimating the ideal frequency for an individual patient, and clinical judgment combined with arterial blood gas measurements is needed (Tables 2 and 3). Use of frequency lower than $3 \mathrm{~Hz}$ is not recommended because the depth of oscillation increases markedly, which may result in increased risk of barotrauma. In addition, the frequency should not be raised higher than $7 \mathrm{~Hz}$ in adults. Frequency is adjusted in $1 \mathrm{~Hz}$ increments based on the $\mathrm{PCO}_{2}$ (see below).

The amount of polarity voltage (also called power, amplitude, or $\Delta \mathrm{P}$ ) applied to the electrical coil determines the distance that the piston is driven toward/away from the patient's airway. Therefore, increasing the polarity voltage increases the piston movement or amplitude. The easiest way to conceptualize this is to view it as the means by which tidal volumes are delivered and removed about the mean airway pressure (Figure 3). The greater the piston displacement, the more volume delivered to the patient. The extent to which the amplitude increases depends on the resistance the piston encounters to forward movement. For example, when the oscillator is used in a patient with low compliance or high airway resistance, the piston meets greater pressure during the inspiratory phase, resulting in lesser change in the effective tidal volume. In addition, as
Table 3. Summary of Important Respiratory Therapy and Nursing Considerations With Regard to HFOV Use and Routine Maintenance

Perform thorough suction before connecting to the oscillator

Perform recruitment maneuver before connecting to the oscillator

Use closed system suction catheter

Avoid disconnection from the ventilator

Check for changes in pitch/rhythm of delivered breaths

Check chest/thigh wiggle and changes in chest/thigh wiggle

Always humidify gases

If oscillator stops during suctioning; silence alarm, pull back catheter and restart oscillator

Obtain blood gases and chest x-ray 2 hours after HFOV commencement and at least daily thereafter

Ensure appropriate education regarding the oscillator for relatives of the patient

NOTE: HFOV = high-frequency oscillatory ventilation.

depicted in Figure 3, expiration is an active process in HFOV. This is because negative displacement of the diaphragm results in subatmospheric pressure. Generally, the starting power setting should be 70 to $90 \mathrm{~cm} \mathrm{H}_{2} \mathrm{O}$. Most commonly, this variable is adjusted to obtain a slight wiggle to the level of the patient's thigh, though it has been recommended that the starting setting be $\mathrm{PCO}_{2}+20 \mathrm{~cm} \mathrm{H}$ O. As described below, subsequent adjustments are made based on the $\mathrm{PCO}_{2}$.

The mean pressure adjust control mechanism allows for adjustments in $\mathrm{P}_{\mathrm{aw}}$. This control varies the resistance placed on a mushroom-shaped control valve on the patient circuit at the terminus of the expiratory limb. Following manual recruitment efforts, increasing the $\mathrm{P}_{\mathrm{aw}}$ keeps alveoli open at a constant pressure, thus minimizing or avoiding 
atelectrauma from shearing forces. As with all forms of mechanical ventilation, increases in mean airway pressure result in enhanced oxygenation. Although increasing $\mathrm{P}_{\mathrm{aw}}$ increases the transpulmonary pressure, it does not affect cardiac output in euvolemic patients. ${ }^{9}$ As discussed below, the mean pressureadjust control is bias flow dependent. Most authors recommend a starting $\mathrm{P}_{\text {aw }} 5 \mathrm{~cm} \mathrm{H}_{2} \mathrm{O}$ above the last plateau pressure noted during conventional ventilation with a maximal starting $\mathrm{P}_{\mathrm{aw}}$ of $35 \mathrm{~cm} \mathrm{H}_{2} \mathrm{O}$. Based on outcome studies on conventional ventilation, the goal is to keep the $\mathrm{P}_{\mathrm{aw}}$ less than $30 \mathrm{~cm} \mathrm{H}_{2} \mathrm{O}$. Mean airway pressure should be adjusted in increments of $2 \mathrm{~cm} \mathrm{H}_{2} \mathrm{O}$ based on the oxygen saturation. Patients who have recurrent hypoxemic events that resolve with recruitment should have their $\mathrm{P}_{\mathrm{aw}}$ increased.

Bias flow is the rate at which gas flows through the ventilator circuit. The generally accepted starting bias flow rate is $40 \mathrm{~L} / \mathrm{min}$, and the maximal flow possible on the $3100 \mathrm{~B}$ is $60 \mathrm{~L} / \mathrm{min}$. An increase in bias gas flow will increase $\mathrm{P}_{\mathrm{aw}}$, thereby improving oxygenation. The maximal flow may be needed to maintain $\mathrm{P}_{\mathrm{aw}}$ in patients with a large air-leak, such as bronchopleural fistulae. However, the maximal flow rate is not sufficient to support significant spontaneous respiratory efforts and is one reason that patients must be deeply sedated or pharmacologically relaxed while on HFOV.

In conjunction with amplitude, mean airway adjust, bias flow, and frequency control, the percentage of inspiratory time can also be adjusted. Because the endotracheal (ET) tube contributes at least $50 \%$ of the total airway resistance during expiration, the inspiratory time setting should always be less than $50 \%$ to minimize the risk of air trapping and voluand barotraumas. ${ }^{16}$ Inspiratory time of $33 \%$ is optimal because it results in a drop in the mean intrapulmonary pressure as a result of higher flow-dependent ET tube resistance during inspiration. This is due to higher flow rates during the shortened inspiratory phase. ${ }^{16}$ Increasing the inspiratory time will improve both oxygen (by increasing $\mathrm{P}_{\mathrm{aw}}$ ) and $\mathrm{CO}_{2}$ exchange (by increasing delivered tidal volume), though it can also increase the risk of lung injury. Because of this, it is the variable least commonly altered to address blood gas values.

\section{Gas Exchange}

Tidal volumes delivered using the $3100 \mathrm{~B}$ are not measured and are estimated to be 1 to $2 \mathrm{~cm}^{3} / \mathrm{kg}-$ approximately the volume of anatomic dead space. There are several mechanisms postulated to explain gas transport under these nonphysiologic conditions (Figure 4), and the reader is referred to more definitive sources for greater detail of information. ${ }^{17,18}$ Briefly, the gas transport in the most proximal airway occurs by convection, and gradually transitions into a mixture of convection and diffusion and finally purely diffusion as one progresses along the airway tree. ${ }^{17}$ Bulk flow can still provide conventional gas delivery to proximal alveoli with low regional dead space volumes. ${ }^{18}$ There is also the presence of coaxial flow, wherein the gas in the center of large airways and the ET tube flows inward while gas on the periphery flows outward. This can develop because of the asymmetric low profile of high velocity gases. ${ }^{18}$ Dispersion phenomena can produce mixing of fresh and residual gas along the flow front of gas through a tube. Pendelluft flow refers to flow of gas between adjacent alveoli with different impedance, as seen in ARDS. ${ }^{17,18}$ Collateral ventilation and cardiogenic mixing also play a role. ${ }^{17}$ Finally, augmented molecular diffusion can occur at the alveolar level secondary to the added kinetic energy from the oscillations. ${ }^{16-18}$ The importance of each of these mechanisms is debated, and it has been suggested that a combination of all the above factors may be in play simultaneously during HFOV. ${ }^{16,17,19}$

As with any form of mechanical ventilation, oxygenation on HFOV can be improved by either increasing the $\mathrm{FiO}_{2}$ or increasing the mean airway pressure. As noted above, the mean airway pressure can be increased directly or it can be adjusted by increasing the inspiratory time. Increases in $\mathrm{P}_{\mathrm{aw}}$ result in greater alveolar recruitment and subsequent improvement in $\mathrm{V} / \mathrm{Q}$ matching. However, increasing $\mathrm{P}_{\mathrm{aw}}$ to a point where perialveolar vessels collapse from alveolar overdistention can result in both seemingly paradoxical hypoxemia and/or barotrauma to the lung. Because $\mathrm{P}_{\mathrm{aw}}$ is affected by many variables, such as inspiratory time or ET cuff leak (discussed below), one must be cognizant of the fact that a change in one variable may affect the other variables and vice versa.

Ventilation on HFOV is facilitated by the extremely efficient mixing of gas in the airways. Carbon dioxide removal is approximately proportional to the product of oscillation frequency and the amplitude squared. ${ }^{20}$ Thus, changes in tidal volume have a greater impact on $\mathrm{CO}_{2}$ clearance than changes in respiratory rate. This explains why decreasing the 


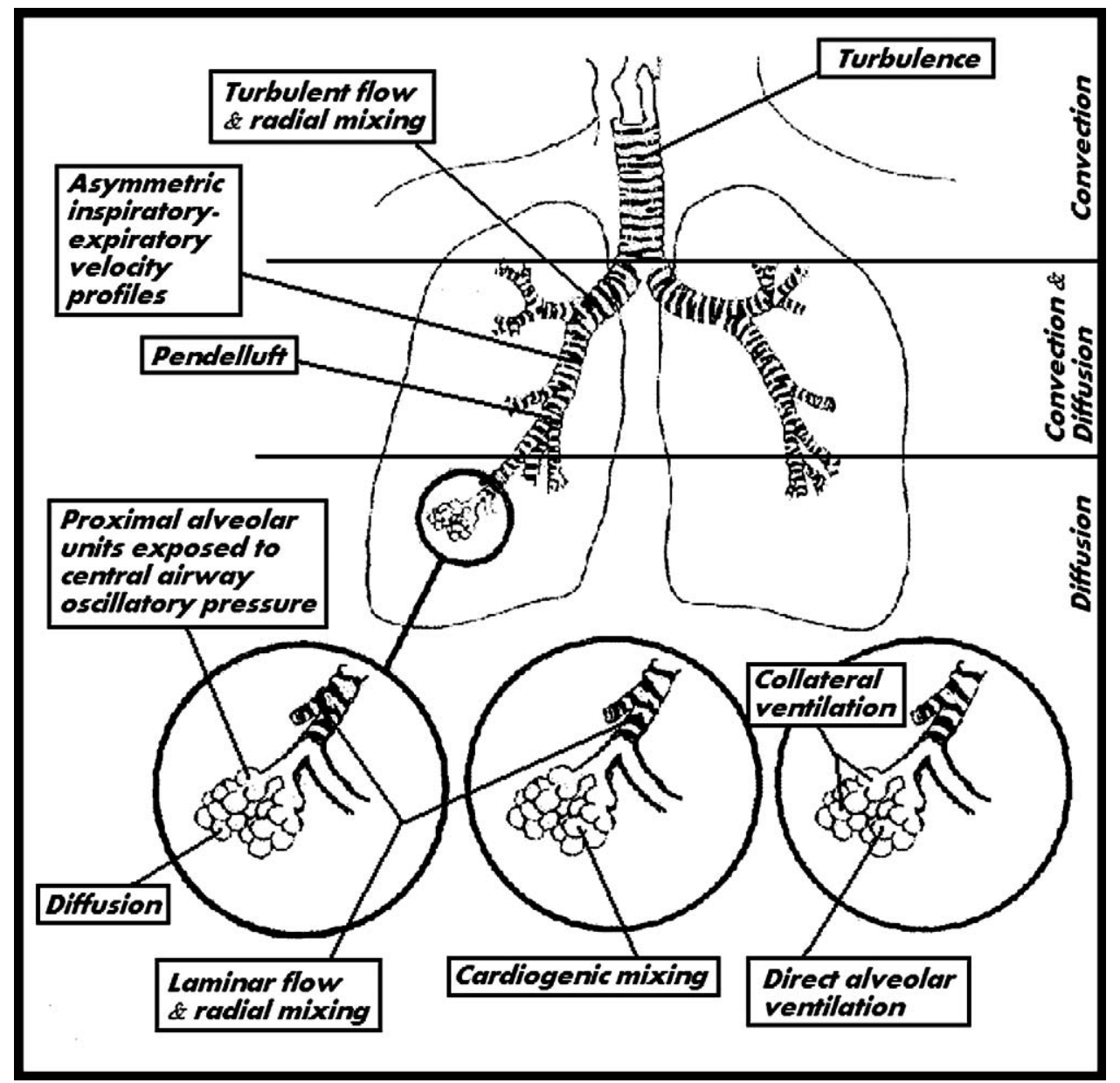

Figure 4. Possible mechanisms to account for gas exchange at various levels of the bronchoalveolar tree during high frequency oscillation.

frequency results in an increase in $\mathrm{CO}_{2}$ clearancethat is, decreasing the frequency results in an increase in delivered tidal volume. Furthermore, even the smallest adjustments in amplitude or changes in lung compliance and delivered tidal volume have a great effect on ventilation. Consequently, $\mathrm{CO}_{2}$ elimination is controlled primarily by adjusting amplitude first and then by adjusting frequency. ${ }^{20}$ Lastly, ventilation can also be augmented by decreasing the anatomic dead space. This can be done by deflating the cuff of the ET tube sufficiently to decrease the $\mathrm{P}_{\text {aw }} 5$ to $8 \mathrm{~cm} \mathrm{H}_{2} \mathrm{O}$ (and compensating for this decrease by manually increasing the $\mathrm{P}_{\mathrm{aw}}$ again). However, this may result in an increased risk of aspiration or derecruitment of the lung, though adverse outcomes have not been reported in studies on adult patients.

Because there is no bulk flow of gas, significant respiratory acidosis is common at the start of HFOV.
Patients who have significant preexisting acidemia may need to be temporized with intravenous buffering solutions while $\mathrm{CO}_{2}$ exchange stabilizes. Table 4 lists the factors associated with decreased tidal volume delivery and thus impaired ventilation with HFOV.

To summarize, oxygenation is improved by increasing mean airway pressure, $\mathrm{FiO}_{2}$, or percentage of inspiratory time. Ventilation $\left(\mathrm{CO}_{2}\right.$ exchange $)$ is improved by decreasing frequency, increasing power, increasing inspiratory time, or creation of a cuff leak. The risks and benefits of changes in each variable have to be considered. Table 2 list suggested ventilator adjustments based on arterial blood gas results.

\section{Interaction With Spontaneous Breathing}

Spontaneous breaths contribute useful lung reexpanding forces. ${ }^{21,22}$ Much like in APRV, spontaneous 
Table 4. Factors Contributing to Decreased Tidal Volume Delivery With HFOV

Decreased endotracheal tube diameter

Mucous plug or alveolar edema fluid accumulation

Decreased ventilator power or amplitude

Decreased percentage of inspired time

Decreased respiratory system compliance

Increased endotracheal tube length

Increased ventilator frequency

NOTE: $\mathrm{HFOV}=$ high frequency oscillatory ventilation.

breaths on HFOV help maintain end-expiratory alveolar expansion in dependent lung regions and improve $\mathrm{V} / \mathrm{Q}$ distributions. ${ }^{23,24} \mathrm{~A}$ significant reduction in days on ventilator was observed when spontaneous breathing efforts were allowed in patients with ALI/ARDS. ${ }^{22}$ However, vigorous spontaneous respiratory efforts, especially in large size patients, may contribute to sudden pressure variations that activate equipment alarms, interrupt oscillations, and produce significant oxygen desaturation. It is for this reason that the early HFOV trials in adults recommended administration of muscular blockade. Current sedation protocols attempt to maintain the patient's ability to breathe spontaneously with small tidal volumes while suppressing deep breaths or coughing, thus minimizing potential complications related to myopathy/neuropathy of prolonged neuromuscular blockade. ${ }^{25,26}$

\section{Weaning From HFOV}

There is no consensus on how to wean patients from HFOV to conventional ventilation. However, a protocol used in 2 randomized trials called for the $\mathrm{P}_{\mathrm{aw}}$ to decrease in increments of $2 \mathrm{~cm} \mathrm{H}_{2} \mathrm{O}$ to a goal of $30 \mathrm{~cm} \mathrm{H}_{2} \mathrm{O}$ after the $\mathrm{FiO}_{2}$ had been weaned to less than $60 \%{ }^{13,14}$ The $\mathrm{FiO}_{2}$ was then weaned to $40 \%$ if the oxygen saturation $\left(\mathrm{SaO}_{2}\right)$ remained greater than $88 \%$. Finally, the $\mathrm{P}_{\text {aw }}$ was weaned again to a final goal of 20 to $25 \mathrm{~cm} \mathrm{H}_{2} \mathrm{O}$. The patient was then transitioned to a lung protective volume-controlled mode of ventilation if the $\mathrm{SaO}_{2}$ remained greater than $88 \%$ for 24 hours. Initial conventional ventilation settings were designed to ensure that the mean airway pressure remained 20 to $25 \mathrm{~cm} \mathrm{H}_{2} \mathrm{O}$. The patient was considered to fail this transition and was converted back to HFOV if the $\mathrm{SaO}_{2}$ decreased to less than $88 \%$ in the first 48 hours following transition. This protocol is supported by other studies that suggest that weaning the $\mathrm{P}_{\mathrm{aw}}$ to less than $20 \mathrm{~cm} \mathrm{H}_{2} \mathrm{O}$ prior to transitioning to conventional ventilation can cause derecruitment of alveoli and hypoxemia. ${ }^{25}$ In instances where the initial $\mathrm{P}_{\mathrm{aw}}$ is greater than $35 \mathrm{~cm}$ $\mathrm{H}_{2} \mathrm{O}$ and the $\mathrm{FiO}_{2}$ is greater than $60 \%$, some authors suggest weaning the $\mathrm{P}_{\text {aw }}$ and $\mathrm{FiO}_{2}$ concurrently to try to minimize excess stretch and barotrauma on the alveoli as quickly as possible. In these instances, the $\mathrm{P}_{\mathrm{aw}}$ should be weaned to $35 \mathrm{~cm} \mathrm{H}_{2} \mathrm{O}$ initially, then the $\mathrm{FiO}_{2}$ should be weaned to less than $60 \%$, and then the protocol outlined above can be followed.

\section{Complications and Drawbacks of HFOV}

Despite research showing benefits of HFOV, use of oscillatory ventilation is rare in adult patients, and comfort level is generally low among medical personnel using this equipment, ${ }^{27}$ consequently resulting in difficulty with troubleshooting and adjusting the ventilator. Possible complications include overdistention/underdistention of the lung, pneumothorax, ET occlusion from secretions, and hemodynamic compromise. Other limitations include inability to transport the patient and deliver nebulized medications.

The possibility of lung overdistention on HFOV due to trapping of gas has been investigated. ${ }^{28}$ Because this cannot be measured directly, the exact extent to which this is a problem is controversial, but there is no difference in the reported rate of pneumothorax between HFOV and conventional ventilation. ${ }^{13}$. Lung underdistention can also be a problem under HFOV. Although controversial, small tidal volumes delivered at a constant mean airway pressure may actually exacerbate or result in progressive atelectasis, one of the problems HFOV is thought to overcome. This underscores the importance of recruiting the lung at the start of HFOV.

Continuous positive intrathoracic pressure impedes venous return to the heart and therefore cardiac output. This can result in hypotension when patients are transitioned from conventional ventilation to HFOV. Patients must be euvolemic prior to initiation of HFOV to minimize this risk, and the bedside practitioner should be ready to volume resuscitate the patient as needed. As previously described, systemic hypotension may also be worsened by transient acidemia.

Metered dose inhalers are largely ineffective during HFOV, with only about $25 \%$ of a nebulized medication being detectable at the end of the ET tube. ${ }^{29}$ 
Better methods to deliver aerosolized medications with HFOV are being developed.

Patient transport during HFOV may be a significant logistical problem because there is no portable version of the equipment. However, an effective protocol for transport of patients on HFOV has been described. ${ }^{7}$ This mechanism involves clamping the ET tube while on HFOV, transition to a self-inflating bag with $20 \mathrm{~cm} \mathrm{H}_{2} \mathrm{O}$ PEEP valve, unclamping of the ET tube with vigorous manual ventilation during transport, and reversal of these procedures once an oscillatory ventilator has been set up at the trip destination. ${ }^{7}$ Recruitment maneuvers can be used as needed to reestablish oxygenation. This protocol notwithstanding transport of such patients is potentially dangerous and should be minimized.

\section{Unique Aspects Regarding HFOV}

There are a number of specific respiratory therapy and nursing aspects that should be highlighted with regards to patients on HFOV (Table 3). The sight of someone being "oscillated" can be disturbing for the family and friends of the patient. Hence, it is important to ensure adequate education regarding expectations provided to the patient's family and friends.

It is difficult to appropriately auscultate the chest while a patient is on HFOV. Physicians and nurses must rely on chest $\mathrm{x}$-ray and objective measures (eg, vital signs, ventilator readings, and blood gas analysis) to detect new lung pathology. Daily chest $\mathrm{x}$-ray is needed to ensure adequate lung expansion.

A closed system suction unit should be used on HFOV because disconnecting the patient to suction can potentially lead to derecruitment. Unless otherwise indicated, suctioning for the first 24 hours is not necessary. When using a closed system suction system, it is important to draw back the suction catheter all the way from the ET tube upon completion. Ideally, the patient should be thoroughly suctioned before HFOV is commenced. Once the patient is oscillated, every attempt must be made to try not to disconnect the patient from the oscillator to prevent derecruitment.

All clinical staff need to be trained in recognition of HFOV-related complications. This is especially important with HFOV because the ventilator is poorly alarmed to alert the bedside staff to possible complications. Clinical assessment and experience are needed to recognize ET tube obstruction (increase in amplitude with an increase in $\mathrm{P}_{\mathrm{aw}}$, decrease in $\mathrm{SpO}_{2}$, and increase in $\mathrm{PCO}_{2}$ ), tension pneumothorax (decrease in $\mathrm{SpO}_{2}$, disparity in the height of the left and right chest walls, and a fall in blood pressure), or pulmonary overdistension (fall in blood pressure, increased central venous pressure, and decreased $\mathrm{SpO}_{2}$ ). Observation of the patient for equal and continuous chest wiggle should be performed upon initiation of HFOV and followed closely thereafter. Chest wiggle diminishes if the ET tube has moved or is obstructed. Chest wiggle on one side only may indicate that the patient has developed a pneumothorax or has a main-stem intubation. Chest wiggle assessments should be performed following any patient repositioning.

\section{Airway Pressure Release Ventilation}

Airway pressure release ventilation represents another open-lung mechanical ventilation strategy. Airway pressure release ventilation was designed to provide the oxygenation benefits of a nearpermanent recruitment maneuver, while augmenting ventilation for patients with low-compliance lung disease. $^{30}$ Stock et $\mathrm{al}^{31}$ were first to describe APRV in 1987 and are credited with its introduction.

Airway pressure release ventilation has been described as continuous positive airway pressure (CPAP) with regular, brief, intermittent releases in airway pressure. ${ }^{30}$ It can therefore be thought of as a time-cycled, pressure-limited mode of mechanical ventilation, which operates by cycling between 2 pressure levels within a high-flow (demand-valve) CPAP circuit that allows spontaneous breathing at any phase of the ventilatory cycle. This mechanism may reduce the need for heavy sedation ${ }^{31-33}$ and requires patient involvement, thus precluding the use of paralysis.

The degree of ventilatory support with APRV is determined by the duration of the 2 CPAP levels and the distending pressure used to recruit alveoli with each mechanical cycle. ${ }^{31,34}$ The tidal volume generated depends mainly on respiratory compliance and the difference between the CPAP levels; because, $\mathrm{P}_{\text {low }}$ is usually set at zero, tidal volume is dependent on $\mathrm{P}_{\text {high. }}$ Of interest, when spontaneous breathing is absent, APRV is indistinguishable from inverse ratio pressure-controlled, time-cycled ventilation. ${ }^{31,34}$ 


\section{Rationale Behind APRV}

Lungs of patients with ALI/ARDS are often characterized by heterogeneity of threshold opening pressures across different lung areas. ${ }^{35}$ The cyclic change in pressure and tidal volume that is characteristic of conventional ventilation may preferentially fill and overdistend alveoli with a short-time constant for filling (ie, nonfibrotic alveoli) while inducing atelectrauma in fibrotic alveoli with a longer time constant for filling. Airway pressure release ventilation aims to minimize this risk by keeping the lung inflated for an extended period of time while minimizing the exhalation (or release) phase.

Compared to conventional ventilation, APRV is associated with significantly lower peak/plateau airway pressures for a given tidal volume. ${ }^{30}$ In a multicenter, prospective crossover trial of patients with ALI, Rasanen et $\mathrm{al}^{36}$ demonstrated a $55 \%$ reduction in peak airway pressures compared with conventional ventilation while maintaining similar oxygenation and ventilation. By keeping the lung expanded for an extended period of time and allowing minimal time for exhalation, APRV may produce nearly complete recruitment while minimizing lower volume-induced lung injury associated with cyclic recruitment and atelectrauma. ${ }^{30}$ However, as opposed to HFOV, APRV allows for a brief exhalation period. It is known that ARDS is a heterogeneous process whereby less fibrotic regions of the lung receive inspiratory flow preferentially while less compliant alveoli collapse earlier during exhalation. Therefore, APRV could theoretically cause VILI by inducing volutrauma during the inhalation phase and atelectrauma during the release phase in patients with ARDS. As discussed below, ventilator adjustments based on the flow-versus-time and pressure-versus-time curves are needed to minimize this risk.

There is evidence that points to important differences between ventilation distribution during spontaneous breathing and controlled mechanical ventilation. ${ }^{37}$ During spontaneous breathing, the posterior muscular sections of the diaphragmatic muscle move more than the anterior tendon plate. Thus, in a supine patient, the dependent portions of the lungs are better ventilated during spontaneous breathing. Because in conventional methods of ventilation the diaphragm remains more passive, and because of the complex interactions between abdominal and thoracic forces, mechanical ventilation tends to be distributed more towards the anterior, nondependent, and relatively less perfused areas of the lung. ${ }^{38}$ Paralysis and heavy sedation further inhibit diaphragmatic contraction leading to cephalad migration and further compression of dorsal inferior lung parenchyma. Therefore, more atelectasis is observed in the dorsal lung areas, which are closer to the diaphragm. By recruiting alveoli and reestablishing functional residual capacity at a more favorable point on the pressure-volume curve, APRV can "unload" inspiratory muscles and decrease the work of breathing associated with acute restrictive lung disease. ${ }^{39}$ Spontaneous breathing, therefore, might require less effort. The ability to have variable gas flow and accommodate spontaneous ventilation is a key difference between HFOV and APRV. Spontaneous breathing with APRV in experimental lung injury models was associated with less atelectasis by computed tomographic evidence. $^{40}$ Furthermore, Neumann et $\mathrm{al}^{41}$ demonstrated that allowing spontaneous breathing with APRV decreased intrapulmonary shunt by increasing ventilation of aerated-dependent lung tissue and opening atelectatic lung parenchyma. ${ }^{41}$

Although relatively weak, there is evidence that use of APRV may be associated with decreases in multiorgan failure and perhaps mortality (when compared to mortality in the ARDSNet trial), ${ }^{4,35}$ as well as significant decrease in the need for sedatives as compared to volume-controlled ventilation in postcardiac surgery patients. ${ }^{42}$ Furthermore, when compared to pressure-controlled ventilation, APRV has been associated with higher cardiac index and lower systemic and pulmonary vascular resistance. ${ }^{33,43}$ In early clinical studies, APRV was demonstrated to be a feasible alternative to conventional mechanical ventilation in patients with ALI of mild-to-moderate severity. ${ }^{36}$

\section{Mechanics of APRV}

The 5 major parameters that need to be adjusted when using APRV are $\mathrm{FiO}_{2}, \mathrm{P}_{\text {high }}$ (high pressure), $\mathrm{T}_{\text {high }}$ (time spent at the high pressure), $\mathrm{P}_{\text {low }}$ (low pressure), and $\mathrm{T}_{\text {low }}$ (time spent at the low pressure). The $P_{\text {high }}$ and $T_{\text {high }}$ are the main determinants of mean airway pressure and thus are directly correlated with oxygenation. ${ }^{35}$ Because mean airway pressure also directly correlates with mean alveolar 


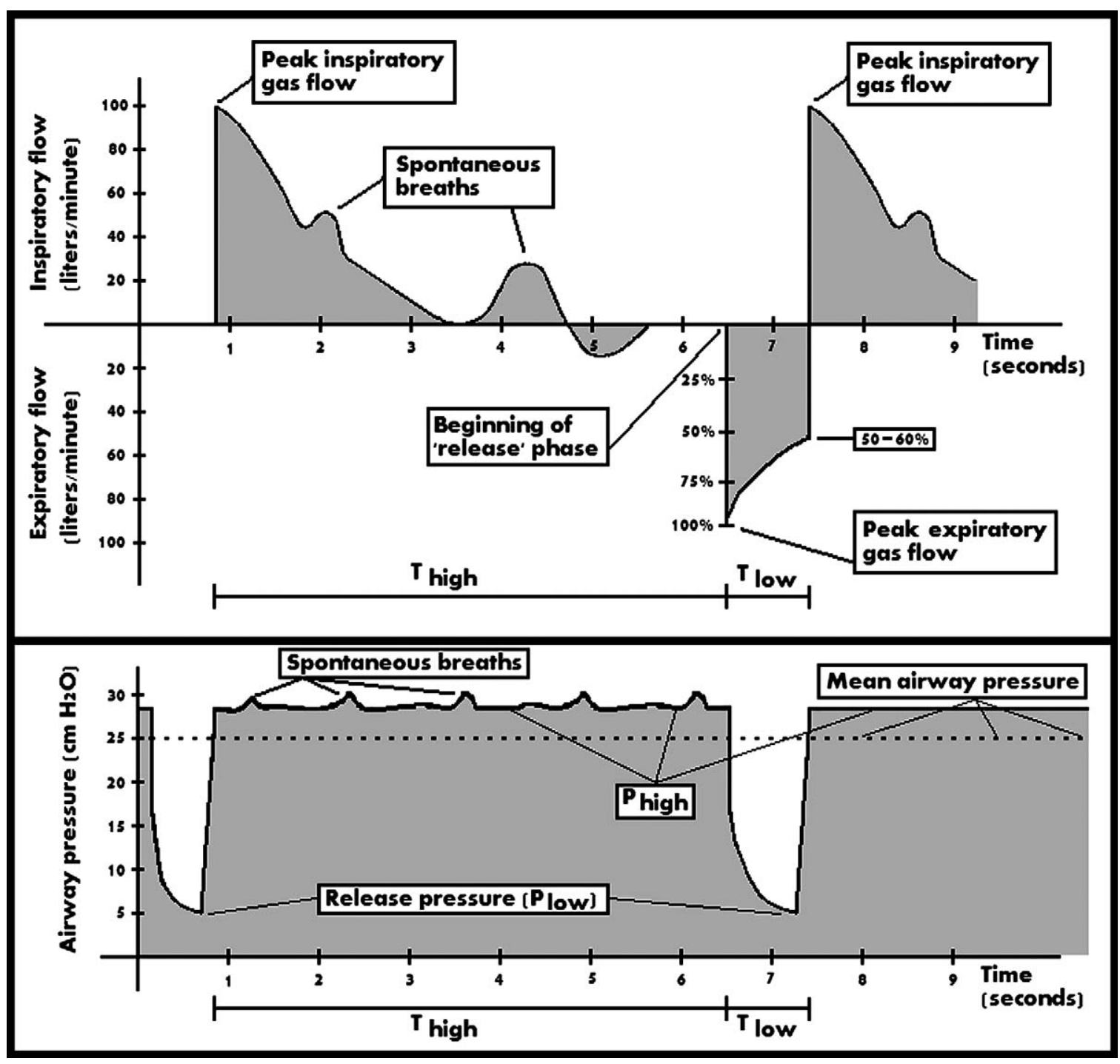

Figure 5. Pressure versus time and flow versus time graphs seen with airway pressure release ventilation. Note that spontaneous breaths are present during the respiratory cycle. Furthermore, note that $\mathrm{T}_{\text {low }}$ is set such that end-expiratory flow is $50 \%$ to $60 \%$ of peak expiratory flow.

volume (thus alveolar-capillary diffusion surface area), $P_{\text {high }}$ and $T_{\text {high }}$ are directly related to gas exchange. The pressure gradient between $\mathrm{P}_{\text {high }}$ and $\mathrm{P}_{\text {low }}, \mathrm{T}_{\text {low }}$, and the patient's spontaneous minute ventilation are the main determinants of alveolar ventilation, thus, $\mathrm{CO}_{2}$ clearance. Suggested initial ventilator settings are noted in Table 1 .

The $\mathrm{P}_{\text {low }}$ and $\mathrm{T}_{\text {low }}$ regulate end-expiratory lung volume and should be optimized to prevent alveolar closure and associated derecruitment while maximizing alveolar ventilation. Generally, the majority $(80 \%-95 \%)$ of the total cycle time $\left(\mathrm{T}_{\text {high }}+\mathrm{T}_{\text {low }}\right)$ should be spent at $\mathrm{P}_{\text {high }}$ to optimize mean alveolar volume and thus maximize potential gas exchange. To minimize derecruitment, the $\mathrm{T}_{\text {low }}$ should be set such that expiration ends when expiratory flow equals $50 \%$ to $75 \%$ of peak expiratory flow rate (PEFR; Figure 5). Because the flow curve shown on the ventilator is a summation of all alveoli, it is possible that severely fibrotic alveoli with a shorttime constant for exhalation will collapse and sustain atelectrauma if exhalation time is extended. Moreover, VILI may be possible even with this recommended exhalation time.

Newly intubated adult patients who are started on APRV should have their $\mathrm{P}_{\text {high }}$ set at the desired plateau 
pressure. If a patient is transitioning from conventional ventilation, their $\mathrm{P}_{\text {high }}$ should be set at their most recent plateau pressure (usually 20-35 $\mathrm{cm} \mathrm{H}_{2} \mathrm{O}$ ). When transitioning from HFOV to APRV, the patient should be placed on $\mathrm{P}_{\text {high }}$ equal to mean $\mathrm{P}_{\text {aw }}$ plus 2 to $4 \mathrm{~cm} \mathrm{H}_{2} \mathrm{O} .{ }^{35}$ Regardless of when the patient was intubated or prior mode of ventilation, $\mathrm{P}_{\text {low }}$ is set at $0 \mathrm{~cm}$ $\mathrm{H}_{2} \mathrm{O}, \mathrm{T}_{\text {high }}$ between 4 and 6 seconds, and $\mathrm{T}_{\text {low }}$ between 0.6 and 0.8 seconds based on the expiratory flow measurements. ${ }^{35}$ Pressure $_{\text {low }}$ is set to zero to optimize the pressure gradient during the release phase, thus maximizing exhalatory flow and alveolar ventilation. Despite the set $P_{\text {low }}$, the release phase $\left(T_{\text {low }}\right)$ is very brief, preventing an airway pressure of zero. Time low $_{\text {ow }}$ is kept brief to prevent actual alveolar collapse or derecruitment. Of note, patients with obstructive lung disease should have their initial $\mathrm{T}_{\text {low }}$ set between 0.8 and 1.5 seconds and adjusted based on the expiratory flow measurements. ${ }^{35}$ In both scenarios, the pressure-time curve can be used to confirm that the airway pressure never reaches zero.

\section{Special Measured Parameters}

The PEFR is the maximal expiratory flow rate achieved during the release phase of APRV. It is a function of the pressure gradient between $\mathrm{P}_{\text {high }}$ and $\mathrm{P}_{\text {low }}$, lung volume, compliance of the lung and thorax, and the airway resistance. Peak expiratory flow rate termination (T-PEFR) is a measured parameter that represents the expiratory flow rate at the end of the release phase of the APRV cycle. Peak expiratory flow rate termination is useful in guiding APRV adjustments, when used as a ratio of PEFR. ${ }^{35}$ To keep things practical, a ratio of T-PEFR to PEFR of $60 \%$ is ideal. However, because of individual variability, a ratio between $50 \%$ and $66 \%$ may be used successfully (Figure 5).

\section{Gas Exchange}

As with HFOV, a large part of gas exchange occurs through convection and diffusion during the inspiratory phase of APRV. Alveolar recruitment results in improved V/Q matching, intrapulmonary shunting, and arterial oxygenation relative to conventional ventilation. ${ }^{23}$ The increase in arterial oxygenation supports the notion of ongoing recruitment of previously nonventilated lung areas, especially when coupled with improved pulmonary compliance. The improvement in oxygenation occurs gradually over a period of 24 hours and results from gradual pulmonary recruitment. ${ }^{44}$

As with all modes of ventilation, oxygenation is increased by increasing the $\mathrm{FiO}_{2}$ or the mean airway pressure. The mean airway pressure in APRV is increased by increasing the $\mathrm{P}_{\text {high }}$ or the $\mathrm{T}_{\text {high }}$. Increasing the $\mathrm{P}_{\text {high }}$ beyond $30 \mathrm{~cm} \mathrm{H}_{2} \mathrm{O}$ risks inducing barotrauma, however, may be indicated in patients with poor thoracic or abdominal compliance. ${ }^{35}$

Airway pressure release ventilation is capable of either augmenting alveolar ventilation in the spontaneously breathing patient or facilitating ventilation in the apneic patient. ${ }^{45}$ In fact, APRV was initially described as an improved method of ventilatory support in the presence of ALI and hypercarbia. ${ }^{31}$ Airway pressure release ventilation augments $\mathrm{CO}_{2}$ removal by improving $\mathrm{V} / \mathrm{Q}$ matching and decreasing intrapulmonary shunting. A limiting factor for carbon dioxide clearance is the gradient between alveolar and arterial $\mathrm{CO}_{2}$. This gradient is refreshed during the "release" phase of APRV when fresh gas is exchanged in the alveolar tree. The optimal release frequency is variable and depends upon a number of factors including $\mathrm{CO}_{2}$ production, V/Q mismatch, shunt, and cardiac output. As noted above, $\mathrm{CO}_{2}$ exchange may decrease as the $\mathrm{T}_{\text {high }}$ increases because the respiratory frequency decreases. ${ }^{35}$ This phenomenon is similar to that observed with decreased $\mathrm{CO}_{2}$ clearance with increasing $\mathrm{I}$ :E ratios.

Although the bulk of $\mathrm{CO}_{2}$ exchange occurs during the release phase, other mechanisms such as cardiogenic mixing and spontaneous breathing also affect on alveolar ventilation. Cardiogenic mixing results in $\mathrm{CO}_{2}$ movement toward central airways during the $\mathrm{T}_{\text {high }}$ (or breath-hold) period, improving the ventilatory effectiveness of the release. ${ }^{46}$ The addition of spontaneous breaths during the $T_{\text {high }}$ period further enhances recruitment and ventilation efficiency. ${ }^{35}$ Manipulation of $\mathrm{T}_{\text {high }}$ must be tailored to each patient because of the number of factors involved with $\mathrm{CO}_{2}$ removal. Table 2 lists recommended ventilator adjustments based on blood gas results.

\section{Weaning From APRV}

Patients with adequate or improving oxygenation while on APRV can be progressively weaned by 
lowering the $\mathrm{P}_{\text {high }}$ and extending the $\mathrm{T}_{\text {high. }}$. As a result, the number of timed releases is decreased and the machine minute ventilation is reduced. This forces the patient to increase spontaneous minute ventilation to maintain constant total minute ventilation. This can be equated to a progressive spontaneous breathing trial and a relatively "smooth" transition toward CPAP. Some authors believe that the ultimate weaning target of APRV is CPAP, and that pressure-assisted breathing (pressure support) may actually be counter-productive. ${ }^{35}$ Patients can be transitioned back to conventional ventilation when the $\mathrm{P}_{\text {high }}$ is less than $20 \mathrm{~cm} \mathrm{H}_{2} \mathrm{O}$, the $\mathrm{T}_{\text {high }}$ is greater than 6 seconds, and the $\mathrm{FiO}_{2}$ is less than $40 \%$. Although it has not been studied, transitioning to conventional ventilation may expedite weaning in an environment where care providers are not as familiar with APRV.

\section{Complications and Drawbacks of APRV}

In general, APRV is not indicated in patients who are not breathing spontaneously. Although APRV can oxygenate adequately in the absence of spontaneous breathing, the patient's spontaneous breaths contribute a significant amount of the total minute ventilation. Because many of the proposed advantages of APRV (improved gas exchange, reduced dead space, possible decreased requirement for sedation and analgesia, and improved hemodynamics) are thought to be due to the preservation of spontaneous breathing, the absence of spontaneous breathing renders benefits of APRV relatively ineffective. ${ }^{47}$

As with HFOV, intravascular volume often needs to be augmented in patients on APRV to offset the decrease in venous return to the heart, which results from prolonged positive intrathoracic pressure. However, as opposed to HFOV, these cardiovascular side effects may be minimized on APRV by reducing mechanical ventilation to a level that provides adequate support for existing spontaneous breathing while avoiding overly high levels of positive airway pressure. ${ }^{48}$ Periodic reduction of intrathoracic pressure achieved by maintaining spontaneous breathing during mechanical ventilation promotes venous return to the heart and increased cardiac output and oxygen delivery. ${ }^{49}$ Moreover, the periodic release of positive intrathoracic pressure also augments venous return to the heart.
Other relative contraindications to APRV include patients with severe obstructive pulmonary conditions who are unable to empty their lungs in less than 2 seconds. ${ }^{18}$ This group includes patients with severe asthma and chronic obstructive pulmonary disease (COPD).

\section{Conclusion}

Both HFOV and APRV have been shown to improve oxygenation in patients with ARDS. The important difference between APRV and HFOV is that APRV allows spontaneous ventilation via an elaborate pressure-release valve mechanism, while HFOV is often incompatible with spontaneous breathing. Consequently, APRV may be associated with reduction in the need for or degree of sedation and therefore may be associated with decreased number of ventilator days. Conversely, HFOV aims to keep the lung open at all times, has less of a theoretical chance of causing VILI by inducing volu- or atelectrauma, and mandates sedation. Lastly, APRV ventilators are battery powered and therefore can be used during patient transport. This can be a distinct advantage for patients who need ongoing intervention or testing. To date, there has not been either equivalency or superiority studies comparing these 2 modalities.

A series of randomized, prospective studies are needed to compare outcomes from use of either modality both as initial therapy for evolving ARDS and as rescue modalities for patients who fail accepted conventional ventilation strategies. It is possible that either APRV or HFOV may be equivalent to (if not superior to) low tidal volume and moderate PEEP ventilation due to the concerns regarding ongoing volu- and atelectrauma described above.

\section{References}

1. Dreyfuss D, Soler P, Basset G, Saumon G. High inflation pressure pulmonary edema. Respective effects of high airway pressure, high tidal volume, and positive end-expiratory pressure. Am Rev Respir Dis. 1988;137(5): 1159-1164.

2. Webb HH, Tierney DF. Experimental pulmonary edema due to intermittent positive pressure ventilation with high inflation pressures. Protection by positive end-expiratory pressure. Am Rev Respir Dis. 1974;110(5):556-565. 
3. Amato MB, Barbas CS, Medeiros DM, et al. Effect of a protective-ventilation strategy on mortality in the acute respiratory distress syndrome. $N$ Engl J Med. 1998; 338(6):347-354.

4. Ventilation with lower tidal volumes as compared with traditional tidal volumes for acute lung injury and the acute respiratory distress syndrome. $N$ Engl J Med. 2000;342(18):1301-1308.

5. Lachmann B. Open up the lung and keep the lung open. Intensive Care Med. 1992;18(6):319-321.

6. Hemmila MR, Napolitano LM. Severe respiratory failure: advanced treatment options. Crit Care Med. 2006;34(9 suppl):S278-S290.

7. Cartotto R, Ellis S, Gomez M, Cooper A, Smith T. High frequency oscillatory ventilation in burn patients with the acute respiratory distress syndrome. Burns. 2004; 30(5):453-463.

8. Simma B, Fritz M, Fink C, Hammerer I. Conventional ventilation versus high-frequency oscillation: hemodynamic effects in newborn babies. Crit Care Med. 2000;28(1):227-231.

9. Fort P, Farmer C, Westerman J, et al. High-frequency oscillatory ventilation for adult respiratory distress syndrome-a pilot study. Crit Care Med. 1997;25(6):937-947.

10. Fessler H, Derdak S, Ferguson N, et al. A protocol for high-frequency oscillatory ventilation in adults: results from a roundtable discussion. Crit Care Med. 2007;35(7): 1649-1654.

11. Bollen CW, van Well GT, Sherry T, et al. High frequency oscillatory ventilation compared with conventional mechanical ventilation in adult respiratory distress syndrome: a randomized controlled trial [ISRCTN24242669]. Crit Care. 2005;9(4):R430-R439.

12. Derdak S, Mehta S, Stewart TE, et al. High-frequency oscillatory ventilation for acute respiratory distress syndrome in adults: a randomized, controlled trial. Am J Respir Crit Care Med. 2002;166(6):801-808.

13. Ferguson ND, Chiche JD, Kacmarek RM, et al. Combining high-frequency oscillatory ventilation and recruitment maneuvers in adults with early acute respiratory distress syndrome: the Treatment with Oscillation and an Open Lung Strategy (TOOLS) Trial pilot study. Crit Care Med. 2005;33(3):479-486.

14. Mehta S, Lapinsky SE, Hallett DC, et al. Prospective trial of high-frequency oscillation in adults with acute respiratory distress syndrome. Crit Care Med. 2001;29(7):1360-1369.

15. Venegas JG, Fredberg JJ. Understanding the pressure cost of ventilation: why does high-frequency ventilation work? Crit Care Med. 1994;22(9 suppl):S49-S57.

16. Pillow JJ, Neil H, Wilkinson MH, Ramsden CA. Effect of I/E ratio on mean alveolar pressure during highfrequency oscillatory ventilation. J Appl Physiol. 1999; 87(1):407-414.
17. Pillow JJ. High-frequency oscillatory ventilation: mechanisms of gas exchange and lung mechanics. Crit Care Med. 2005;33(3 suppl):S135-S141.

18. Weavind L, Wenker O. Newer modes of ventilation: an overview. Int J Anesthesiol. 2000;4:4.

19. MacIntyre NR. High-frequency ventilation. Crit Care Med. 1998;26(12):1955-1956.

20. Keszler M. High frequency ventilation: evidence-based practice and specific clinical indications. Neo Reviews. 2006;7(5):e234-e49.

21. Putensen C, Hering R, Wrigge H. Controlled versus assisted mechanical ventilation. Curr Opin Crit Care. 2002;8(1):51-57.

22. Putensen $\mathrm{C}$, Zech S, Wrigge $\mathrm{H}$, et al. Long-term effects of spontaneous breathing during ventilatory support in patients with acute lung injury. Am J Respir Crit Care Med. 2001;164(1):43-49.

23. Putensen C, Mutz NJ, Putensen-Himmer G, Zinserling J. Spontaneous breathing during ventilatory support improves ventilation-perfusion distributions in patients with acute respiratory distress syndrome. Am J Respir Crit Care Med. 1999;159(4 pt 1):1241-1248.

24. Wrigge H, Zinserling J, Neumann P, et al. Spontaneous breathing improves lung aeration in oleic acid-induced lung injury. Anesthesiology. 2003;99(2):376-384.

25. Derdak S. High-frequency oscillatory ventilation for acute respiratory distress syndrome in adult patients. Crit Care Med. 2003;31(4 suppl):S317-S323.

26. Higgins J, Estetter B, Holland D, Smith B, Derdak S. High-frequency oscillatory ventilation in adults: respiratory therapy issues. Crit Care Med. 2005;33(3 suppl): S196-S203.

27. Duval EL, Markhorst DG, Gemke RJ, van Vught AJ. High-frequency oscillatory ventilation in pediatric patients. Neth J Med. 2000;56(5):177-185.

28. Boros SJ, Mammel MC, Coleman JM, et al. Neonatal high-frequency jet ventilation: four years' experience. Pediatrics. 1985;75(4):657-663.

29. Lowson SM. Inhaled alternatives to nitric oxide. Crit Care Med. 2005;33(3 suppl):S188-S195.

30. Frawley PM, Habashi NM. Airway pressure release ventilation: theory and practice. AACN Clin Issues. 2001;12(2):234-246; quiz 328-329.

31. Stock MC, Downs JB, Frolicher DA. Airway pressure release ventilation. Crit Care Med. 1987;15(5):462-466.

32. Seymour CW, Frazer M, Reilly PM, Fuchs BD. Airway pressure release and biphasic intermittent positive airway pressure ventilation: are they ready for prime time? J Trauma. 2007;62(5): 1298-1308; discussion 308-309.

33. Kaplan LJ, Bailey H, Formosa V. Airway pressure release ventilation increases cardiac performance in patients with acute lung injury/adult respiratory distress syndrome. Crit Care. 2001;5(4):221-226. 
34. Baum M, Benzer H, Putensen C, Koller W, Putz G. Biphasic positive airway pressure (BIPAP) - a new form of augmented ventilation. Anaesthesist. 1989;38(9):452-458.

35. Habashi NM. Other approaches to open-lung ventilation: airway pressure release ventilation. Crit Care Med. 2005;33(3 suppl):S228-S240.

36. Rasanen J, Cane RD, Downs JB, et al. Airway pressure release ventilation during acute lung injury: a prospective multicenter trial. Crit Care Med. 1991;19(10):1234-1241.

37. Froese AB, Bryan AC. Effects of anesthesia and paralysis on diaphragmatic mechanics in man. Anesthesiology. 1974;41(3):242-255.

38. Reber A, Nylund U, Hedenstierna G. Position and shape of the diaphragm: implications for atelectasis formation. Anaesthesia. 1998;53(11):1054-1061.

39. Petrof BJ, Legare M, Goldberg P, Milic-Emili J, Gottfried SB. Continuous positive airway pressure reduces work of breathing and dyspnea during weaning from mechanical ventilation in severe chronic obstructive pulmonary disease. Am Rev Respir Dis. 1990;141(2): 281-289.

40. Wrigge H, Zinserling J, Hering R, et al. Cardiorespiratory effects of automatic tube compensation during airway pressure release ventilation in patients with acute lung injury. Anesthesiology. 2001;95(2):382-389.

41. Neumann P, Wrigge H, Zinserling J, et al. Spontaneous breathing affects the spatial ventilation and perfusion distribution during mechanical ventilatory support. Crit Care Med. 2005;33(5):1090-1095.

42. Rathgeber J, Schorn B, Falk V, Kazmaier S, Spiegel T, Burchardi H. The influence of controlled mandatory ventilation (CMV), intermittent mandatory ventilation (IMV) and biphasic intermittent positive airway pressure
(BIPAP) on duration of intubation and consumption of analgesics and sedatives. A prospective analysis in 596 patients following adult cardiac surgery. Eur J Anaesthesiol. 1997;14(6):576-582.

43. Hering $R$, Peters D, Zinserling J, Wrigge $H$, von Spiegel T, Putensen C. Effects of spontaneous breathing during airway pressure release ventilation on renal perfusion and function in patients with acute lung injury. Intensive Care Med. 2002;28(10): 1426-1433.

44. Sydow M, Burchardi H, Ephraim E, Zielmann S, Crozier TA. Long-term effects of two different ventilatory modes on oxygenation in acute lung injury. Comparison of airway pressure release ventilation and volume-controlled inverse ratio ventilation. Am J Respir Crit Care Med. 1994;149(6):1550-1556.

45. Garner W, Downs JB, Stock MC, Rasanen J. Airway pressure release ventilation (APRV). A human trial. Chest. 1988;94(4):779-781.

46. Engel LA, Menkes H, Wood LD, Utz G, Joubert J, Macklem PT. Gas mixing during breath holding studied by intrapulmonary gas sampling. J Appl Physiol. 1973;35(1):9-17.

47. Branson RD, Johannigman JA. What is the evidence base for the newer ventilation modes? Respir Care. 2004; 49(7):742-760.

48. Kirby RR, Perry JC, Calderwood HW, Ruiz BC, Lederman DS. Cardiorespiratory effects of high positive end-expiratory pressure. Anesthesiology. 1975;43(5): 533-539.

49. Downs JB, Douglas ME, Sanfelippo PM, Stanford W, Hodges MR. Ventilatory pattern, intrapleural pressure, and cardiac output. Anesth Analg. 1977;56(1):88-96. 